\title{
Stem cell transplant for lymphoma - never too late?
}

\author{
Amanda F. Cashen and Nancy L. Bartlett \\ Washington University School of Medicine and Siteman Cancer Center, St. Louis, MO, USA \\ E-mail: AMANDA CASHEN - acashen@wustl.edu
}

doi:10.3324/haematol.2021.280519

In $\mathrm{n}$ this issue, using the Center for International Blood and Marrow Transplant Research (CIBMTR) registry, Mei et al. ${ }^{1}$ provide a retrospective outcomes analysis of 285 patients with relapsed or refractory (R/R) diffuse large B-cell lymphoma (DLBCL) who underwent autologous hematopoietic cell transplantation (autoHCT) after achieving complete (CR) or partial response (PR) to their third or higher line of chemotherapy. Sixty three percent of patients had primary refractory disease or relapsed within 1 year of initial diagnosis. For perspective, during the same interval 577 patients with R/R DLBCL in the registry underwent autoHCT after second line therapy. Patients in the registry with detailed data including prior lines of therapy comprise approximately $8-10 \%$ of all patients in the CIBMTR registry, which in other analyses have been representative of the entire dataset (personal communication with corresponding author). Mei et al. report 5-year overall survival (OS) and progression-free survival (PFS) for the study population of $51 \%$ and $38 \%$. As expected, patients in CR at the time of transplant had better OS and PFS, but a substantial minority of patients with PR also benefited from autoHCT, with a 5-year PFS of $34 \%$. Relapses in patients with a PR occurred early, with a near plateau in the relapse/progression curve after 1 year. In contrast, patients in CR had a significantly lower 1-year risk of relapse but a continuous risk of relapse for at least 4 years after autoHCT, resulting in a non-significant difference in the 5 -year risk of relapse between the two groups (CR 45\% vs. PR 54\%, $P=0.14$ ).

Importantly, while nearly half of all patients were without relapse at 5 years, the 5 -year PFS of $38 \%$ reflects a 5 year non-relapse mortality of $12 \%$. After lymphoma, the second most common cause of death was second malignancy, in $10 \%$ of the study cohort during the entire follow-up period.

How does this retrospective registry analysis inform our decision whether to pursue autoHCT for a given patient with R/R DLBCL? Primarily, it confirms that patients with chemosensitive disease can achieve longterm remissions after autoHCT, including those who have early failure of frontline immunochemotherapy, those who achieve less than a CR to salvage chemotherapy, and those who have been treated with more than one line of salvage chemotherapy. Consistent with prior reports, Mei et al. found that, while patients in CR have a better PFS than those in PR, approximately a third of PR patients remain alive and progression-free after autoHCT. Their results mirror another CIBMTR analysis ${ }^{2}$ that reported 5-year PFS of $41 \%$ for patients with R/R DLBCL who had PET-positive PR prior to autoHCT. Even patients with primary refractory disease or early relapse have a $40-50 \%$ chance of durable PFS after autoHCT, if they respond to salvage therapy., ${ }^{3,4}$

Unfortunately, many patients with R/R DLBCL do not achieve a $\mathrm{CR}$ or $\mathrm{PR}$ to one or more lines of salvage chemotherapy. In the prospective, randomized CORAL study, ${ }^{4} 63 \%$ of all patients, $51 \%$ of patients previously treated with rituximab, and $46 \%$ of patients with primary refractory disease or early relapse achieved CR or PR to RICE or R-DHAP. Of the patients who did not respond to the study-assigned salvage regimen, only $32 \%$ responded to subsequent therapy and underwent a stem cell transplant. ${ }^{6}$ In two randomized studies of anti-CD19 chimeric antigen receptor $\mathrm{T}$-cell therapy (CAR-T) versus standard of care in patients with early failure of frontline immunochemotherapy, the response rates to standard second line therapies were $50 \%{ }^{7}$ and $43 \% .{ }^{8}$ Clearly, inadequate response to second- or third-line therapy is a significant barrier to use of autoHCT in many patients with R/R DLBCL. New agents with encouraging activity in $\mathrm{R} / \mathrm{R}$ DLBCL, including bispecific T-cell engagers ${ }^{9}$ and antibody-drug conjugates, ${ }^{10}$ may improve the number of patients who achieve PR or CR to second- or third-line therapy making autoHCT an option for more patients. However, whether response to novel therapies with unique mechanisms of action will predict response to autoHCT is unknown and will be extremely challenging to study.

While CAR-T is the obvious choice for patients who do not respond to standard second- or third-line regimens, the choice between autoHCT and CAR-T can be difficult for patients with chemosensitive R/R DLBCL. The potential approval of CAR-T in the second line will increase the challenge of weighing the relative benefits of autoHCT versus CAR-T for a given patient. Now more than 4 years after approval of the first CAR-T for R/R DLBCL, significant hurdles remain, including complicated and delayed insurance approvals, limitations on manufacturing slots, and the potential for progressive disease and worsening performance status during the "waiting period". Rigorous cost-benefit analysis of CAR-T versus autoHCT for patients with chemosensitive disease in second and third line would be informative. Additionally, late effects of CAR-T and autoHCT need to be compared and considered in the treatment decision. Post-CAR-T complications, especially prolonged cytopenias and infections, can be more challenging than post-autoHCT in a significant minority of patients, and, as Mei's study demonstrates, second malignancies are a concerning cause of nonrelapse mortality post-autoHCT.

Although we have ample data on the tolerability and efficacy of CAR-T following relapse after autoHCT, there is no data on autoHCT following CAR-T cell failure. In the current use of CAR-T for chemorefractory disease or relapse after autoHCT, patients would not be candidates for autoHCT after CAR-T. However, if approvals are forthcoming for CAR-T as second-line therapy, understanding the feasibility and efficacy of autoHCT after CAR-T would help inform the decision of sequencing these therapies in patients responding to second- or third- 
line chemotherapy. In the meantime, the retrospective studies by Mei et al. and others provide important benchmarks of outcomes following autoHCT, suggesting that autoHCT remains a reasonable option for the patients with chemosensitive R/R DLBCL, even in later lines of therapy. For those patients, CAR-T can be held in reserve in case of relapse after transplant.

\section{Disclosures}

$N L B$ has received personal research funding from KITE/Gilead, has received research funding to the institution from ADC Therapeutics, Bristol-Meyers Squibb, Celgene, Forty Seven, Immune Design, Janssen, KITE Pharma, Merck, Millennium, Pharmacyclics,Pfizer, Roche/Genentech and SeaGen, and sits on the advisory boards of ADC Therapeutics, Roche/Genentech, SeaGen. AFC sits on the advisory board of Secura Bio and has received research funding Secura Bio.

\section{Contributions}

$A C$ and NLB contributed equally to the writing of this editorial.

\section{References}

1. Mei M, Hamadani M, Ahn K, et al. Autologous hematopoietic cell transplantation in diffuse large B-cell lymphoma after 3 or more lines of prior therapy: evidence of durable benefit. Haematologica. 2022;105(5):1214-1217.
2. Shah N, Ahn K, Litovich C, et al. Is autologous transplant in relapsed DLBCL patients achieving only a PET+ PR appropriate in the CAR T-cell era? Blood. 2021;137(10):1416-1423.

3. Bal S, Costa L, Suater C, Litovich C, Hamadani M. Outcomes of autologous hematopoietic cell transplantation in diffuse large B cell lymphoma refractory to firstline chemoimmunotherapy. Transplant Cell Ther. 2021;27(1):55.e1-55.e7.

4. Gisselbrecht C, Glass B, Mounier N, et al. Salvage regimens with autologous transplantation for relapsed large B-cell lymphoma in the rituximab era. J Clin Oncol. 2010;28(27):4184-4190.

5. Hamadani M, Parameswaran N, Zhang Y, et al. Early failure of frontline rituximab-containing chemo-immunotherapy in diffuse large B cell lymphoma does not predict futility of autologous hematopoietic cell transplantation. Biol Blood Marrow Transplant. 2014;20 (11):1729-1736.

6. Van Den Neste E, Schmitz N, Mounier N, et al. Outcome of patients with relapsed diffuse large B-cell lymphoma who fail second-line salvage regimens in the international CORAL study. Bone Marrow Transplant. 2016;51(1):51-57.

7. Locke F, Miklos D, Jacobson C, et al. Axicabtagene ciloleucel as second-line therapy for large B-cell lymphoma. N Eng J Med. 2022;386 (7):640-654.

8. Bishop M, Dickinson M, Purtill D, et al. Second-line tisagenlecleucel or standard care in aggressive B-cell lymphoma. N Eng J Med. 2022;386(7):629-639

9. Budde L, Assouline S, Sehn L, et al. Single-agent mosunetuzumab shows durable complete responses in patients with relapsed or refractory B-cell lymphomas: phase I dose-escalation study. J Clin Oncol. 2022;40(5):481-491

10. Caimi P, Ai W, Alderuccio J, et al. Loncastuximab tesirine in relapsed or refractory diffuse large B-cell lymphoma (LOTIS-2): a multicentre, open-label, single-arm, phase 2 trial. Lancet. 2021;22 (6):790-800. 